\title{
FPGA-Based Approach to Level-1 Track Finding at CMS for the HL-LHC
}

\author{
Louise Skinnari ${ }^{1, a}$ for the CMS Collaboration \\ ${ }^{1}$ Cornell University, Ithaca, NY, USA
}

\begin{abstract}
The high luminosity upgrade of the LHC is expected to deliver luminosities of $7.5 \times 10^{34} \mathrm{~cm}^{-2} \mathrm{~s}^{-1}$, with an average of 140-200 overlapping proton-proton collisions in each bunch crossing at a frequency of $40 \mathrm{MHz}$. To maintain manageable trigger rates under these conditions track reconstruction will be incorporated in the all-hardware first level of the CMS trigger. A track-finding algorithm based on seed tracklets has been developed and implemented on commercially available FPGAs for this purpose. An overview of the algorithm is presented, results are shown of its expected performance from simulations, and an implementation of the algorithm in a Xilinx Virtex-7 FPGA for a hardware demonstrator system is discussed.
\end{abstract}

\section{Introduction}

The upgrade to the High Luminosity LHC (HL-LHC) is planned for 2024-2025 and is expected to deliver a peak luminosity of about $7.5 \times 10^{34} \mathrm{~cm}^{-2} \mathrm{~s}^{-1}$ with the goal of delivering $3000 \mathrm{fb}^{-1}$ per experiment over a ten-year period [1]. The high instantaneous luminosity is predicted to yield an average number of overlapping proton-proton collisions per bunch crossing (pileup) of between 140 and 200. These extreme pileup (PU) conditions create a challenging environment for the LHC experiments and place stringent requirements on the detectors' trigger systems. The CMS [2] physics program for the HL-LHC requires maintaining manageable trigger rates with low thresholds similar to those achieved during Run 1. An integral part of achieving this goal is the addition of track reconstruction in the allhardware Level-1 (L1) trigger. A $40 \mathrm{MHz}$ silicon-based track trigger on the scale of the CMS detector has never been built and it is therefore essential to demonstrate the feasibility of such a system.

The tracklet approach for track reconstruction at L1 is a road-search algorithm, implemented using commercially available field-programmable gate array (FPGA) technology. The ever-increasing capability and the programming flexibility make FPGAs ideal for performing fast track finding. The tracklet approach allows a naturally pipelined implementation with low time multiplexing (typically a factor of 4-8). It also allows for a simple emulation of the algorithm for the full detector.

These proceedings discuss the motivation for incorporating tracking at L1 and give an overview of the CMS track-trigger concept. The tracklet algorithm is described in detail and results of the estimated performance of the algorithm, based on simulation studies in $\mathrm{C}++$ and Verilog, are presented. The development of a hardware demonstrator system using Xilinx Virtex-7 FPGAs is also discussed.

ae-mail: louise.skinnari@cern.ch 


\section{Motivation for L1 tracking}

At the HL-LHC, L1 trigger rates for single muons, electrons, and jets will exceed the current frontend capabilities. Significantly increasing the trigger thresholds would limit the physics potential and would alone be insufficient. The addition of a L1 track trigger is an integral part of maintaining the physics acceptance at the HL-LHC. Including tracking in the L1 trigger allows for improved lepton identification and momentum measurements, the addition of track isolation, and performing vertex identification for hadronic triggers. These additional handles yield large potential reductions of the L1 trigger rates. One example is illustrated in Figure 1, which shows the efficiency for a single muon trigger with $p_{\mathrm{T}}>20 \mathrm{GeV} / c$ as a function of the simulated muon $p_{\mathrm{T}}$ (left) and the trigger rate as a function of the muon $p_{\mathrm{T}}$ threshold (right). The red curves show the efficiencies and rates with the stand-alone L1 muon trigger system for $|\eta|<1.1$ (filled circles) and $1.1 \leq|\eta| \leq 2.4$ (open boxes). The black curves show the efficiencies and rates when incorporating tracking at L1. With the stand-alone muon trigger, low- $p_{\mathrm{T}}$ muons can be misassigned as a high- $p_{\mathrm{T}}$ muon, causing a flattening of the trigger rate with increasing $p_{\mathrm{T}}$ threshold. Using L1 tracking yields an improved momentum measurement and a sharp turn-on at the threshold, which in turn translates to a reduced trigger rate [3].
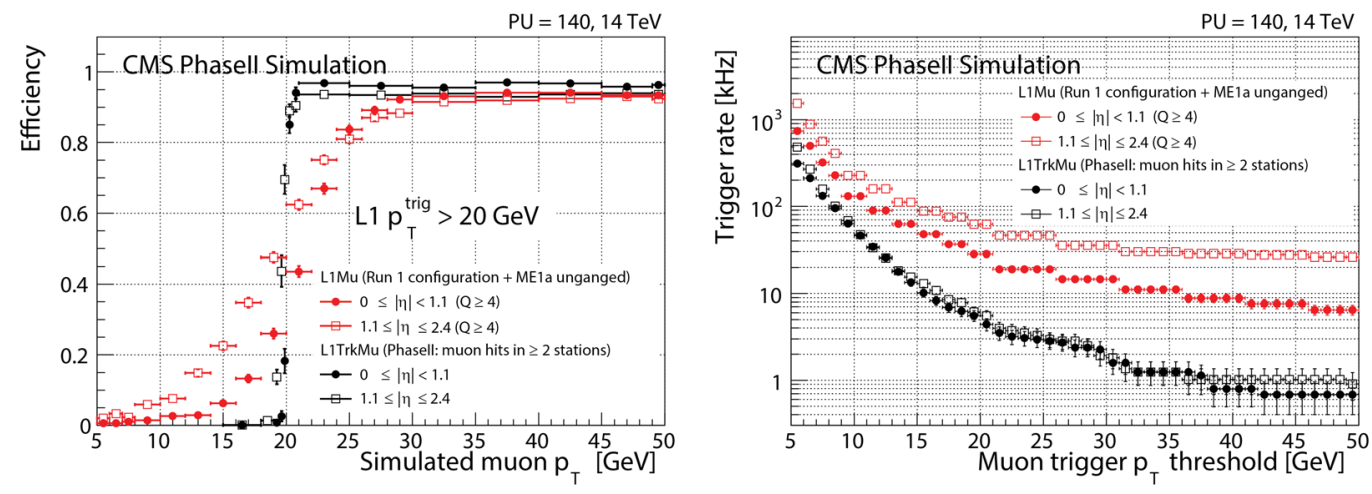

Figure 1: The efficiency of a single muon trigger with $p_{\mathrm{T}}>20 \mathrm{GeV} / c$ as a function of the simulated muon $p_{\mathrm{T}}$ (left) and the trigger rate as a function of the muon trigger threshold (right), shown for the stand-alone muon trigger (red) and when including L1 tracking (black) for $|\eta|<1.1$ (filled circles) and $1.1 \leq|\eta| \leq 2.4$ (open boxes) [3].

\section{Track trigger concept}

The upgraded all-silicon tracker consists of an outer tracker with a central barrel and two endcaps as well as an inner pixel detector, which is not part of the L1 track trigger. Unique to the new outer tracker is the use of " $p_{\mathrm{T}}$ modules" that provide $p_{\mathrm{T}}$ discrimination already in the front-end readout electronics through hit correlations between two closely spaced sensors. Two different module types are employed: pixel-strip (PS) modules in the inner barrel layers and inner half of the disks, and strip-strip (2S) modules in the outer barrel layers and outer half of the disks [3]. The $2 \mathrm{~S}$ modules are composed of two superimposed strip sensors of about $10 \times 10 \mathrm{~cm}^{2}$, mounted with the strips parallel to one another. The PS modules have two sensors of about $5 \times 10 \mathrm{~cm}^{2}$, where one sensor is segmented in strips and the other is composed of $10 \mu \mathrm{m} \times 1.5 \mathrm{~mm}$ "macro-pixels". The pixelated sensor provides 
a precise measurement of the $z$ coordinate, which in turn enables primary vertex discrimination at L1. The output from the PS and 2S modules are correlated pairs of clusters, referred to as "stubs", that are required to be consistent with a $p_{\mathrm{T}}>2 \mathrm{GeV} / c$ track. The stubs are the input to the back-end processing system that performs the $\mathrm{L} 1$ track finding. Since only $\sim 5 \%$ of tracks in minimum-bias events have $p_{\mathrm{T}}>2 \mathrm{GeV} / c$, forming stubs results in a significant rate reduction already for the L1 track input data.

\section{Tracklet approach}

The goal of the L1 track finding is to reconstruct the trajectories of charged particles with $p_{\mathrm{T}}>$ $2 \mathrm{GeV} / c$ and to identify the track $z_{0}$ position (the $z$ coordinate where the track intercepts the $z$-axis) with about $1 \mathrm{~mm}$ precision, similar to the average vertex separation in events with an average pileup of 140. The proposed tracklet method forms track seeds (tracklets) from pairs of stubs in adjacent layers or disks of the outer tracker. The tracklets provide roads where compatible stubs are included to form track candidates. A linearized $\chi^{2}$ fit determines the final track parameters.

The tracklet algorithm has been optimized to provide full coverage of the tracker with the least amount of redundancy in data duplication. The system is divided into 28 sectors in the $r$ - $\phi$-plane, where the processing for each sector is done by a single FPGA. This division limits the need for board-to-board communication to the nearest neighbor on each side.

\subsection{Algorithm overview}

The tracklet algorithm begins with a seeding step where tracklets are formed from pairs of stubs in adjacent layers or disks. An initial estimate of the tracklet parameters is calculated from the two stubs and using the detector origin as a constraint in the $r-\phi$-plane. A tracklet must be consistent with having $p_{\mathrm{T}}>2 \mathrm{GeV} / c$ and $\left|z_{0}\right|<15 \mathrm{~cm}$. The seeding is performed multiple times to ensure good coverage for the full $\eta$ range and for redundancy in the system. The current implementation performs seeding between barrel layers $1+2,3+4$, and $5+6$, between disk $1+2$ and $3+4$, and between barrel layer $1+$ disk 1. The tracking efficiency for different seeding combinations is shown for single muons in Figure 2, using an integer-based $\mathrm{C}++$ emulation of the algorithm as it would be implemented on a FPGA.

The tracklets are projected to other layers and disks to search for matching stubs. The projections use predetermined search windows, derived from residuals between projected tracklets and stubs. The tracklets are projected both inside-out and outside-in. If a matching stub is found, the stub is included in the track candidate and the difference between the projected tracklet and the stub position is stored. A linearized $\chi^{2}$ fit is performed for stubs matched to the trajectory. The track fit implementation uses precalculated derivatives and the tracklet-stub position differences from the projection step. The linearized $\chi^{2}$ fit corrects the initial tracklet parameters to give the final track parameters $p_{\mathrm{T}}, \eta, \phi_{0}, z_{0}$ (and optionally $d_{0}$ ).

A given track can be found many times due to seeding in multiple pairs of layers. Duplicate tracks are removed by comparing tracks in pairs, counting the number of independent and shared stubs. Figure 3 shows for single-muon events the number of tracks found per event prior to any duplicate removal, after duplicate removal between tracks within a $\phi$ sector, and after removing duplicates also between neighboring sectors.

\subsection{Performance studies}

The estimated performance of the tracklet algorithm is studied with a $\mathrm{C}++$ simulation of the algorithm using floating-point precision in the calculations. The L1 tracking efficiency as a function of $\eta$ and 


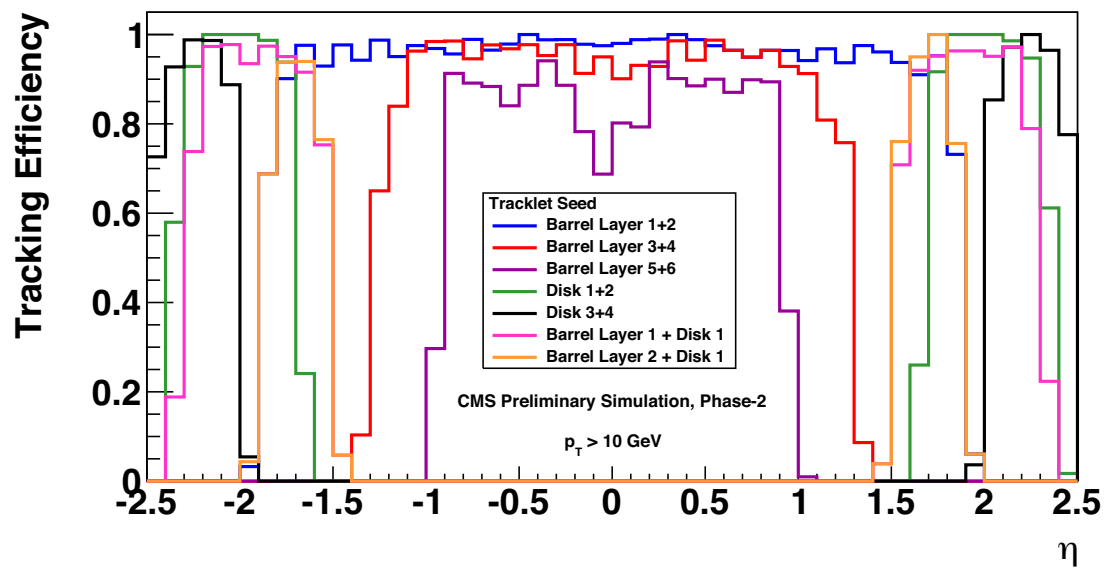

Figure 2: Tracking efficiency for single muons with $p_{\mathrm{T}}>10 \mathrm{GeV} / c$ in events without pileup, shown as a function of muon $\eta$ for different layer and disk combinations in forming the seed tracklets.

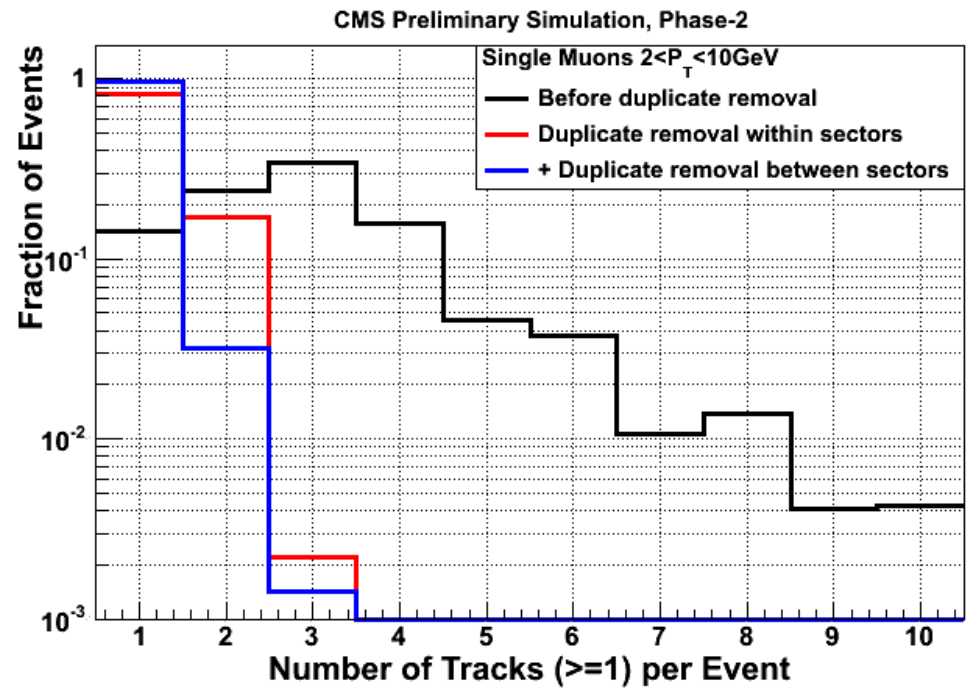

Figure 3: Number of tracks per event for single muons with $2<p_{\mathrm{T}}<10 \mathrm{GeV} / c$ in events without pileup. The number of tracks are shown before any duplicate removal (black) and after duplicate removal within sectors (red), or after also removing duplicates between neighboring sectors (blue). 
$p_{\mathrm{T}}$ is shown in Figure 4 for muons, pions, and electrons in events with $<\mathrm{PU}>=140$. For $|\eta|<1.0$ and $p_{\mathrm{T}}>2 \mathrm{GeV} / c$, an efficiency of $>99 \%, 90 \%$, and $89 \%$ is observed for muons, pions, and electrons, respectively. Pions and electrons have a lower efficiency compared to muons due to their additional interactions with the detector material.
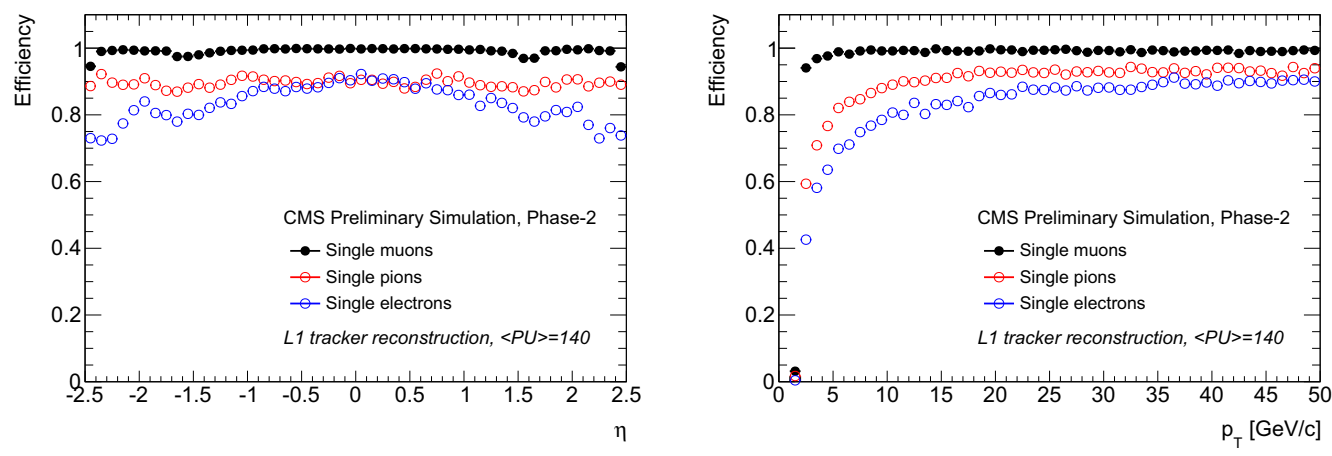

Figure 4: Efficiency for L1 track reconstruction as a function of $\eta$ (left) and $p_{\mathrm{T}}$ (right) for muons, pions, and electrons in events with $<\mathrm{PU}>=140$ [3].

The $\mathrm{L} 1$ track $z_{0}$ resolution and the relative resolution in $p_{\mathrm{T}}$ are shown in Figure 5 for single muons as a function of $|\eta|$ for three different ranges of $p_{\mathrm{T}}$. The $z_{0}$ resolution is about $1 \mathrm{~mm}$, similar to the average separation of pileup vertices, for a wide range of $|\eta|$. The momentum resolution is about $1 \%$ for high- $p_{\mathrm{T}}$ tracks for $|\eta|<1.5$. For higher values of $|\eta|$ the momentum resolution decreases due to the increased extrapolation distance to the beam axis, where the track parameters are calculated. The precise $z_{0}$ resolution allows the selection of tracks originating from a common vertex for use in L1 trigger algorithms and the accurate $p_{\mathrm{T}}$ resolution results in sharp muon trigger thresholds.
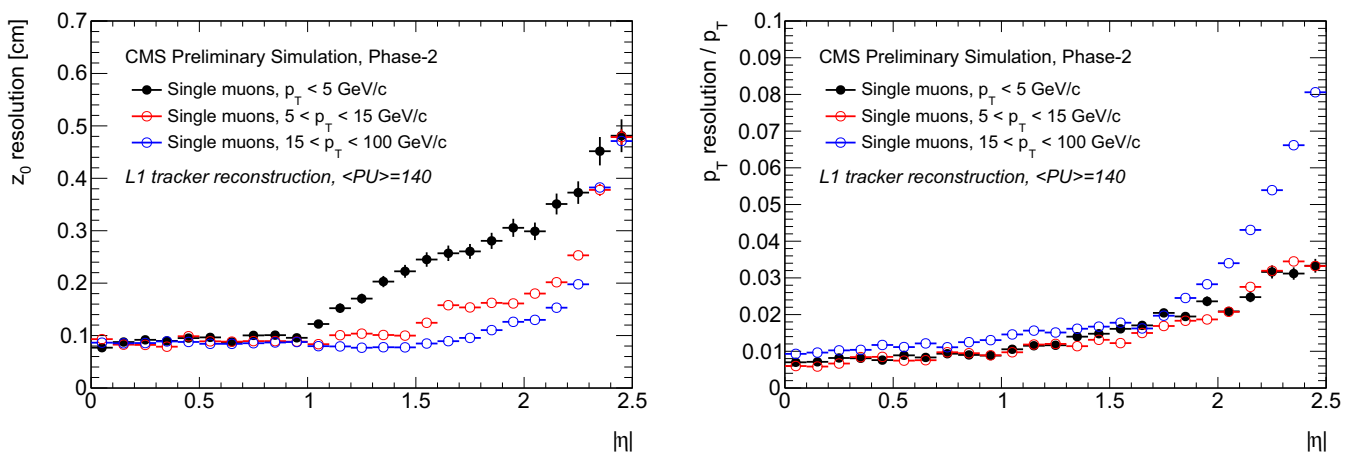

Figure 5: Resolution in $z_{0}$ and relative resolution in $p_{\mathrm{T}}$ for the $\mathrm{L} 1$ track reconstruction of single muons in events with $<\mathrm{PU}>=140$, shown as a function of $|\eta|$ for different $p_{\mathrm{T}}$ ranges [3]. 
The tracklet algorithm has been fully implemented both using floating-point precision in the calculations, as shown above, and as an integer-based $\mathrm{C}++$ emulation of the algorithm as it would be implemented on a FPGA. The floating-point simulation and the integer-based emulation are in good agreement. As an example, track parameter resolutions are compared between the floating-point simulation and the integer-based emulation in Figure 6 for muons with $5<p_{\mathrm{T}}<10 \mathrm{GeV} / c$ and $1.2<|\eta|<2.4$. The root mean square (RMS) of each distribution is calculated. The resolutions as obtained with the integer-based emulation and the (exact) floating-point simulation are compared using $\left(\mathrm{RMS}_{\text {integer }}-\mathrm{RMS}_{\text {float }}\right) / \mathrm{RMS}_{\text {float }}$, calculated to be $0.08,0.10,0.12$, and 0.13 for the relative $p_{\mathrm{T}}$, $\phi, \eta$, and $z_{0}$ resolution, respectively, demonstrating a good level of agreement.

\section{Hardware system}

The primary challenges in building a L1 track trigger system are the large data throughput from the detector at $40 \mathrm{MHz}$ and the short time available to reach a trigger decision. The upgraded L1 trigger will have an overall latency, i.e. the maximum time during which events can be stored in on-detector pipeline memories, of $12.5 \mu \mathrm{s}$, where $4 \mu \mathrm{s}$ are allocated to performing the $\mathrm{L} 1$ track finding.

To address these challenges, the tracklet hardware configuration relies on parallel data processing. The detector is divided into sectors in the $r$ - $\phi$-plane. The current baseline uses $28 \phi$ sectors, so that tracks with $p_{\mathrm{T}}>2 \mathrm{GeV} / c$ span maximally two sectors. This limits the need for data transfer between sectors to the nearest neighbor on each side. A dedicated processing board is used for each sector.

To increase the time available for data processing, the system is time multiplexed. The current baseline assumes a time-multiplex factor of six, so that each copy of the system receives a new event every $150 \mathrm{~ns}$ for a $40 \mathrm{MHz}$ bunch crossing frequency. A different time-multiplex factor of e.g. four or eight (receiving a new event every 100 or $200 \mathrm{~ns}$ ) could also be considered in optimizing for latency or available processing time. The tracklet formation is performed within sectors and a small amount of data is duplicated in every other layer to avoid gaps. Tracklets that project to a neighboring sector are sent there for tracklet-stub matching. By construction, the system operates with a fixed latency.

\subsection{Firmware implementation}

The tracklet algorithm is implemented in firmware as eight processing steps and two transmission steps [4]:

- Stub organization: (1) Sort the input stubs by their corresponding layer, and (2) into smaller units in $z$ and $\phi$, forming what are referred to as "virtual modules".

- Tracklet formation: (3) Select possible tracklets from allowed stub pairs using lookup tables, and (4) calculate the initial tracklet parameters.

- Projections: (5) Transmission of projections pointing to neighboring sectors. (6) Route the projections based on smaller units in $z$ and $\phi$.

- Stub matching: (7) Match projected tracklets to stubs, and (8) calculate the difference in position between the stubs and projected tracklet. (9) Transmission of matches between sectors.

- Track fit: (10) Perform track fit, correcting the initial tracklet parameter estimate.

With a time-multiplex factor of six, a new event is received every $150 \mathrm{~ns}$ at which point the previous event moves to the following processing step. The number of operations performed at each step, such as the number of stub pairs that can be tried in the formation of a valid tracklet, will depend on the clock speed at which the project can run with the hardware used. 
CMS Preliminary Simulation, Phase-2

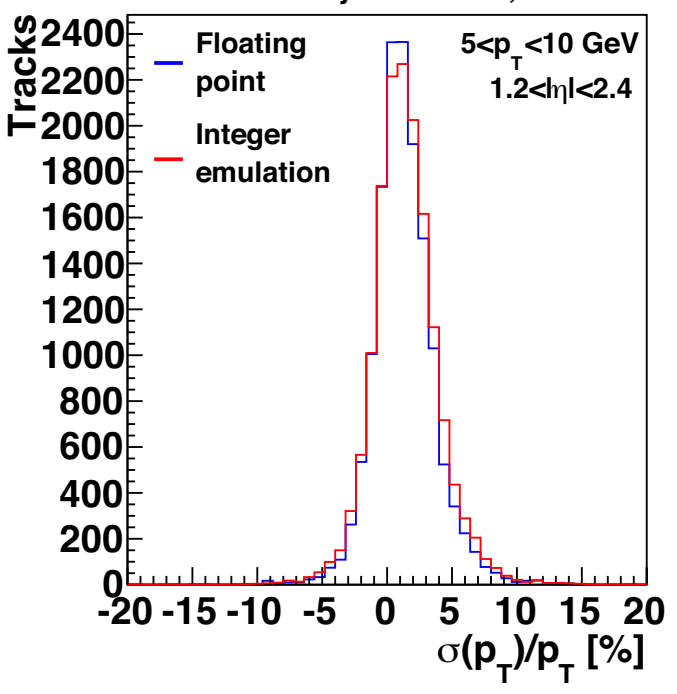

CMS Preliminary Simulation, Phase-2

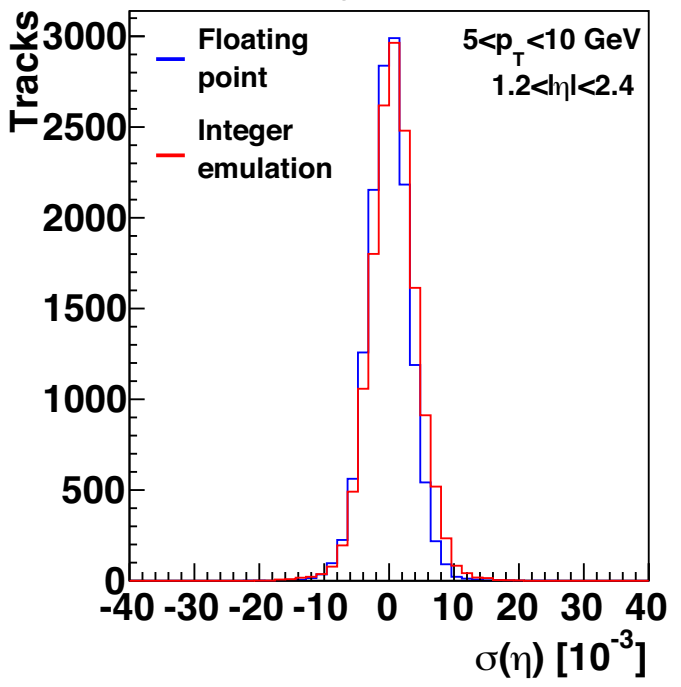

CMS Preliminary Simulation, Phase-2

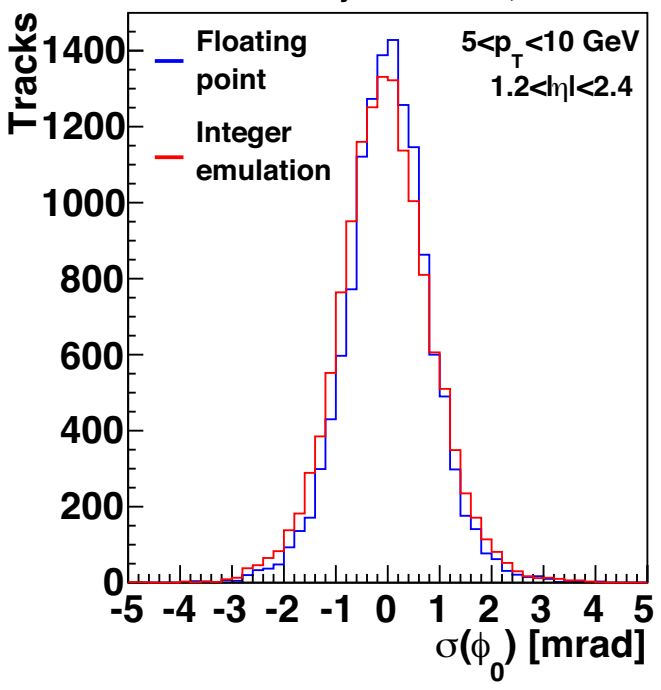

CMS Preliminary Simulation, Phase-2

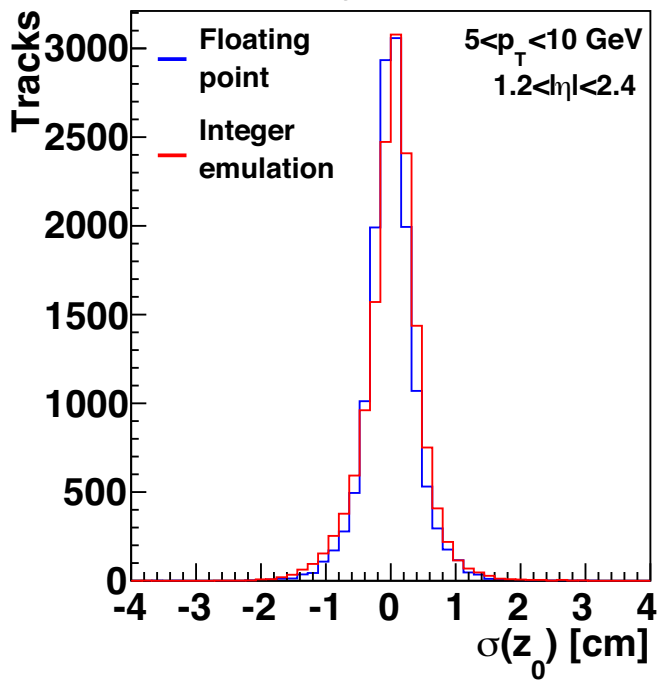

Figure 6: Relative resolution in $p_{\mathrm{T}}$ (top left), resolution in $\phi_{0}$ (top right), $\eta$ (bottom left), and $z_{0}$ (bottom right), for muons with $5<p_{\mathrm{T}}<10 \mathrm{GeV} / c$ and $1.2<|\eta|<2.4$ in events without pileup. 


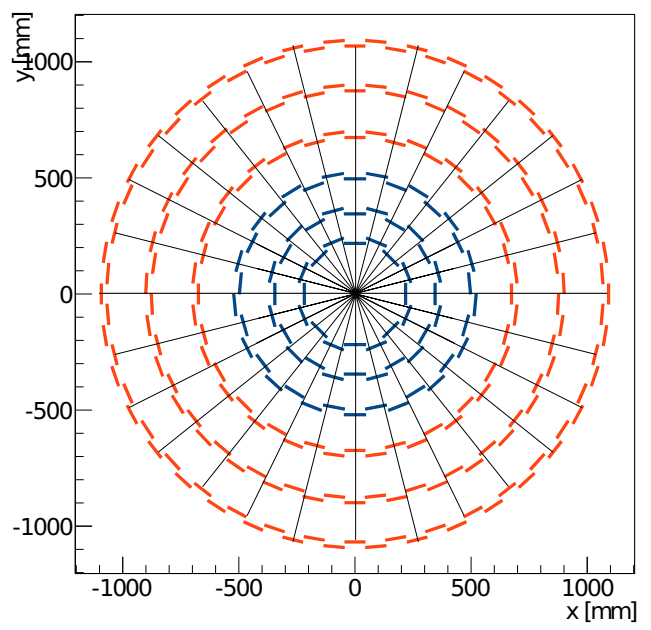

Figure 7: Schematic view in the $r$ - $\phi$-plane of the upgraded outer tracker, dividing into $28 \phi$ sectors so that a track with $p_{\mathrm{T}}>2 \mathrm{GeV} / c$ spans maximally two sectors, limiting the communication between sectors. PS modules (blue) and $2 \mathrm{~S}$ modules (red) are used in the three inner and outer layers, respectively.

The combinatorics in forming tracklets and matching projections to stubs is efficiently reduced by dividing sectors into smaller units in $z$ and $\phi$ to allow additional parallel processing. These smaller units are, as mentioned above, referred to as "virtual modules". Only a small fraction of virtual module pairs can form a valid tracklet - the majority would be inconsistent with a track with $p_{\mathrm{T}}>2 \mathrm{GeV} / c$ and $\left|z_{0}\right|<15 \mathrm{~cm}$. This subdivision efficiently reduces the number of combinations that need to be tried by the algorithm already before doing any event processing.

As the system operates at fixed latency, a given processing step can perform a fixed number of operations. Initial studies of the impact of truncation, such as if there are more stub pairs to try than can be processed within the time constraints of the tracklet formation step, show a robustness of the algorithm to these effects. This is achieved through the use of virtual modules, and the redundancy in using multiple seeding pairs.

The tracklet algorithm including the track fit has been implemented in firmware for half the barrel $(z>0)$ for one sector in $\phi$. Work is ongoing to expand the project to include the forward disks. The tracklet algorithm in the disks is already fully implemented as an integer-based emulation. While the duplicate removal algorithm has not yet been incorporated into the main tracklet project, it is fully implemented in the floating-point simulation, the integer-based emulation, and as a standalone algorithm in firmware on a VC709 evaluation board with a Virtex-7 FPGA [5].

\subsection{Hardware demonstrator}

A system hardware demonstrator has been set up and is now being used for testing the tracklet project. The goal of the demonstrator is to show that the full tracking chain meets the required performance within the available latency. The demonstrator includes three $\phi$ sectors and one time-multiplexing 
slice. A total of four boards are used, one for the central $\phi$ sector, two for its nearest neighbors, and one board that is shared for sending the stub input and receiving the track output.

The boards used for the demonstrator system are $\mu$ TCA boards with a Xilinx Virtex-7 (XC7VX690T) FPGA [5] and a Xilinx Zynq processor for outside communication. These so-called CTP7 boards [6] were developed for the current CMS L1 trigger upgrade [7]. An AMC13 [8] card provides the central clock distribution. The inter-board communication uses $8 \mathrm{~b} / 10 \mathrm{~b}$ encoding with $10 \mathrm{Gbit} / \mathrm{s}$ link speed. The demonstrator system and a CTP7 board are shown in Figure 8.
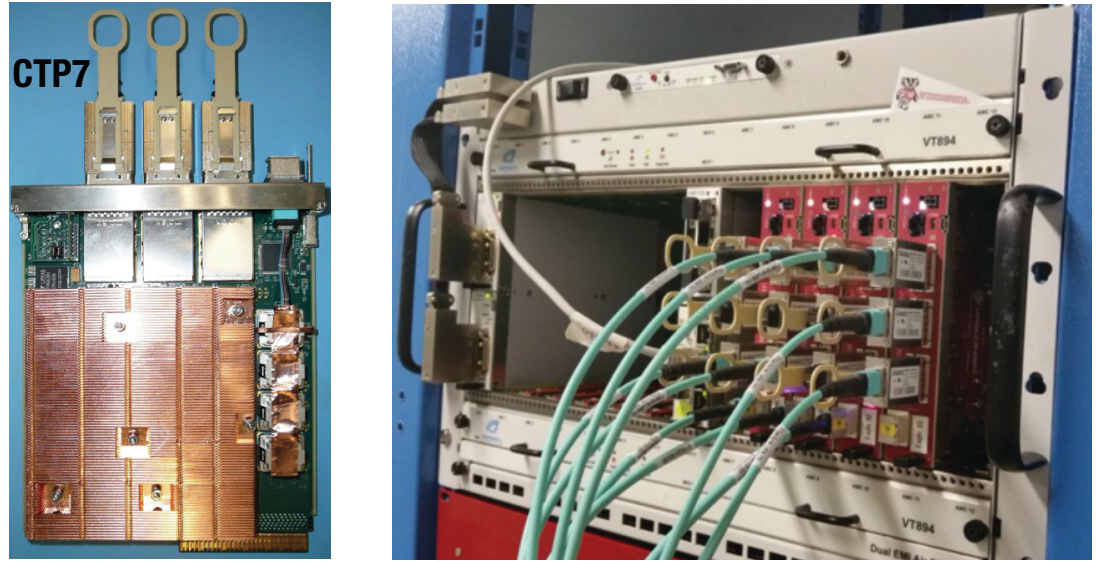

Figure 8: A CTP7 board (left) and the tracklet demonstrator system (right).

One $\phi$ sector for a half-barrel has been implemented on the CTP7 boards, running at $240 \mathrm{MHz}$. Events are processed through the demonstrator as illustrated in Figure 9. First, input stubs obtained from simulations are written to the data trigger and control (DTC) emulator board. On a GO signal, stubs are sent to the three sector processor boards. A new event is sent to each sector board every $150 \mathrm{~ns}$. The events are processed through the sector boards and projections and matches are sent to and received from neighboring boards as required. The final output tracks are received by the track sink board. Systematic studies are performed to compare the integer-based emulation of the tracklet algorithm with a simulation of the firmware using Vivado, as well as with the output tracks from the demonstrator system. Full agreement is observed in processing single-track events between the emulation, firmware simulation, and board output. Studies of many-track events with high pileup are ongoing.

\section{Conclusions}

CMS will require a new all-silicon tracking system with triggering capabilities for the HL-LHC in order to maintain high trigger efficiencies and manageable rates without large increases in trigger thresholds. The tracklet approach is one of the proposed methods for performing L1 track finding. The method is based on a road-search algorithm and uses commercially available FPGA technology. The tracklet algorithm is implemented both as a floating-point simulation and an integer-based emulation of the algorithm as implemented in an FPGA. Half of the barrel has been implemented for a sector in $\phi$ in a Virtex-7 FPGA. The final system, which will not be built for many years, will use futuregeneration (more powerful) FPGAs. To demonstrate the system feasibility, a hardware demonstrator 


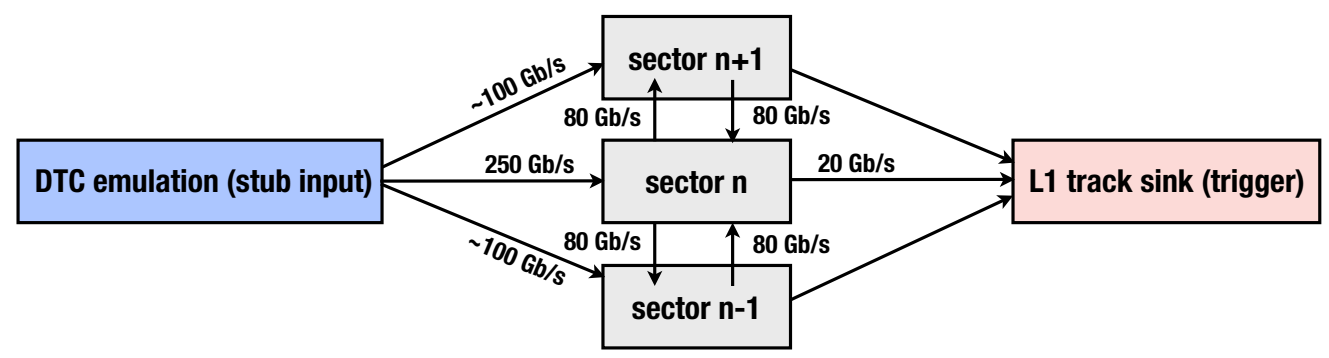

Figure 9: Schematic overview of the tracklet demonstrator system. The DTC emulator and L1 track sink use a single board.

system based on Virtex-7 FPGAs has been assembled and is currently being used to validate the algorithm and board-to-board communication, to measure timing and latency, and to establish the algorithm performance. Preliminary studies from the demonstrator, processing events from the input stubs to the final output tracks, show promising results for the feasibility of the system within the available latency.

\section{Acknowledgements}

This work was supported by the US National Science Foundation through the grant NSF-PHY1307256 . I also wish to thank my tracklet colleagues at Cornell, Rutgers, Ohio State, Notre Dame, and UC Riverside.

\section{References}

[1] F. Zimmermann, PoS EPS-HEP 2009, 140 (2009), https : //cds . cern . ch/record/1211584

[2] The CMS Collaboration, JINST 3, S08004 (2008)

[3] The CMS Collaboration, Tech. Rep. CERN-LHCC-2015-010, CERN, Geneva, Switzerland (2015), https: //cds. cern.ch/record/2020886

[4] J. Chaves, JINST 9, C10038 (2014)

[5] Xilinx Incorporated, 7 Series FPGAs Overview, last accessed on 2016-07-21, pdf

[6] A. Svetek et al., JINST 11, C02011 (2016)

[7] The CMS Collaboration, Tech. Rep. CERN-LHCC-2013-011, CERN, Geneva, Switzerland (2013), https://cds . cern.ch/record/1556311

[8] Boston University, The AMC13 Project, last accessed on 2016-07-21, pdf 